\title{
Differential Gene Expression of Medullary Thyroid Carcinoma Reveals Specific Markers Associated with Genetic Conditions
}

\author{
Agnieszka Maliszewska, ${ }^{\star}$ Luis J. Leandro-Garcia, ${ }^{*}$ Esmeralda Castelblanco, ${ }^{\dagger}$ Anna Macià, ${ }^{\ddagger}$ Aguirre de Cubas, ${ }^{*}$ \\ Gonzalo Goméz-López, ${ }^{\S}$ Lucía Inglada-Pérez, ${ }^{*}$ " Cristina Álvarez-Escolá," Leticia De la Vega, ${ }^{*}$ Rocío Letón, ${ }^{*}$ \\ Álvaro Gómez-Graña, ${ }^{*}$ Iñigo Landa, ${ }^{*}$ Alberto Cascón, ${ }^{*}$ Cristina Rodríguez-Antona, ${ }^{*}{ }^{\boldsymbol{\Phi}}$ Salud Borrego, ${ }^{\boldsymbol{\Phi}}{ }_{* *}$ Mariangela Zane, ${ }^{\dagger \dagger}$ \\ Francesca Schiavi, ${ }^{\ddagger \ddagger}$ Isabella Merante-Boschin, ${ }^{\dagger \dagger}$ Maria R. Pelizzo, ${ }^{\dagger \dagger}$ David G. Pisano, ${ }^{\ddagger}$ Giuseppe Opocher, ${ }^{\dagger \dagger \neq \ddagger}$ \\ Xavier Matias-Guiu, ${ }^{\dagger}$ Mario Encinas, ${ }^{* \ddagger}$ and Mercedes Robledo*ף
}

\begin{abstract}
From the Hereditary Endocrine Cancer Group* Human Cancer Genetics Program, and the Bioinformatics Unit, ${ }^{\S}$ Structural Biology Program, Spanish National Cancer Research Center (CNIO), Madrid, Spain; the Department of Pathology and Molecular Genetics, ${ }^{\dagger}$ Arnau de Vilanova University Hospital, Lleida, Spain; the Neuronal Signaling Unit, ${ }^{\ddagger}$ Department of Basic Medical Sciences, School of Medicine, University of Lleida and the Biomedical Research Institute of Lleida (IRBLleida), Lleida, Spain; the Center for Biomedical Network Research on Rare Diseases (CIBERER), "Valencia, Spain; the Endocrinology Service, "La Paz University Hospital, Madrid, Spain; the Genetics, Reproduction, and Fetal Medicine Clinical Management Unit, ${ }^{* *}$ Institute of Biomedicine of Seville (IBIS), Virgen del Rocío University Hospital, the Spanish National Research Council (CSIC), and the University of Seville, Seville, Spain; the Department of Medicine DIMED,$^{\dagger \dagger}$ University of Padua, Padua, Italy; and the Familial Cancer Clinic and Endocrine Oncology, ${ }^{\ddagger \ddagger}$ Veneto Institute of Oncology, Padua, Italy
\end{abstract}

Accepted for publication October 31, 2012.

Address correspondence to Mercedes Robledo, Ph.D., Hereditary Endocrine Cancer Group, Human Cancer Genetics Program, Centro Nacional de Investigaciones Oncológicas, Melchor Fernández Almagro 3, 28029 Madrid, Spain, or to Mario Encinas, Ph.D., Neuronal Signaling Unit, Universitat de Lleida/Institut de Recerca Biomèdica de Lleida, Lleida, Spain. E-mail: mrobledo@cnio. es or mario.encinas@mex.udl. cat.

\begin{abstract}
Medullary thyroid carcinoma accounts for $2 \%$ to $5 \%$ of thyroid malignancies, of which $75 \%$ are sporadic and the remaining $25 \%$ are hereditary and related to multiple endocrine neoplasia type 2 syndrome. Despite a genotype-phenotype correlation with specific germline RET mutations, knowledge of pathways specifically associated with each mutation and with non-RET-mutated sporadic MTC remains lacking. Gene expression patterns have provided a tool for identifying molecular events related to specific tumor types and to different clinical features that could help identify novel therapeutic targets. Using transcriptional profiling of 49 frozen MTC specimens classified as RET mutation, we identified PROM1, LOXL2, GFRA1, and $D K K 4$ as related to $R E T^{M 918 T}$ and GAL as related to $R E T^{634}$ mutation. An independent series of 19 frozen and 23 formalin-fixed, paraffin-embedded (FFPE) MTCs was used for validation by RT-qPCR. Two tissue microarrays containing 69 MTCs were available for IHC assays. According to pathway enrichment analysis and gene ontology biological processes, genes associated with the MTC ${ }^{\text {M918T }}$ group were involved mainly in proliferative, cell adhesion, and general malignant metastatic effects and with Wnt, Notch, NFKB, JAK/ Stat, and MAPK signaling pathways. Assays based on silencing of PROM1 by siRNAs performed in the MZ-CRC-1 cell line, harboring RET ${ }^{M 918 T}$, caused an increase in apoptotic nuclei, suggesting that PROM1 is necessary for survival of these cells. This is the first report of $P R O M 1$ overexpression among primary tumors. (Am J Pathol 2013, 182: 350-362; http://dx.doi.org/10.1016/j.ajpath.2012.10.025)
\end{abstract}

Supported in part by the Fundación Mutua Madrileña (project AP2775/2008 to M.R.), Fondo de Investigaciones Sanitarias (project PI080883 and PI11/01359 to M.R.), the Spanish Ministry of Science and Innovation (MICINN; BFU2010-17628 to M.E.), and by grants 2009SGR794, RD06/0020/1034, and the programa de intensificación de la investigación, Instituto Carlos III). A.M. and A.d.C. are predoctoral fellows of the "la Caixa" Spanish National Cancer Research Center (CNIO) international Ph.D. program. L.I.-P. is a predoctoral fellow of the CIBERER. L.J.L.-G. is a predoctoral fellow of the Fondo de Investigaciones Sanitarias. E.C. holds a predoctoral fellowship from the
Fundació Alicaia Cuello de Merigó and the Agency for Management of University and Research Aid (Agència de Gestió d'Ajuts Universitaris i de Recerca; AGAUR 2010FI-B01057). Tumor samples were obtained with the support of the Spanish National Tumor Bank Network (Red Nacional de Biobancos; RD09/0076/00047), the Biobank of the Virgen del Rocío Hospital (RD09/0076/00085), the Surgical Pathology Department of Medical and Surgical Sciences of Padua, the Catalan Tumor Bank Network (Xarxa Catalana de Bancs de Tumors), the Tumor Bank Platform of the Network for Cooperative Cancer Research (RTICC), and the Arnau de Vilanova Hospital. 
Medullary thyroid carcinoma (MTC) is a rare malignancy derived from calcitonin-producing parafollicular thyroid cells (thyroid C cells); it constitutes $5 \%$ to $10 \%$ of all thyroid neoplasias. ${ }^{1}$ Approximately $25 \%$ of all MTCs are hereditary, as part of multiple endocrine neoplasia type 2 (MEN2; OMIM \#171400), a rare cancer syndrome $(1: 30,000)$ that follows an autosomal dominant inheritance pattern and exhibits a variable clinical expression.

MEN2 is subdivided into MEN2A, MEN2B, and familial medullary thyroid carcinoma (FMTC), according to the patient's clinical features. Specific activating point mutations in the RET proto-oncogene are responsible for the three forms of MEN2. RET encodes a receptor tyrosine kinase (RTK) implicated in neural crest tissue development and differentiation, ${ }^{2}$ that activates a variety of signaling pathways. Ret signaling takes place mainly through autophosphorylation of Ret tyrosine residues, which trigger the recruitment of several intercellular adapters upon phosphorylation, leading to the activation of several cascades.

Individuals with MEN2A commonly carry RET mutations in codon 634 encoding cysteine, ${ }^{3}$ and less frequently in the cysteine-encoding codons $609,611,618,620$, and $630 .{ }^{4,5}$ Those with a mutated codon 634 have a high risk of lymph node metastasis and a more than $40 \%$ increased risk of developing the disease by age $20 .{ }^{6}$ Individuals with FMTC carry mainly RET mutations affecting highly conserved cysteine regions, such as codons $609,611,618$, and 620 , as well as other infrequent mutations in residues of the intracellular domain, such as in exons $13^{7}$ and $15 .{ }^{8} R E T^{M 918 T}$ mutation in exon 16 correlates with aggressive disease ${ }^{9-11}$ and is found as a germline mutation in the majority of MEN2B cases (94\%) and as a somatic mutation in $30 \%$ to $50 \%$ of sporadic MTC cases. ${ }^{11}$ This mutation leads to constitutive activation of RET and transforming potential in a ligand-independent manner. ${ }^{12}$

MTC progresses slowly and metastasizes to cervical and mediastinal nodal groups in up to $50 \%$ of cases. It also metastasizes to distant organs such as lungs, liver, and bones in $20 \%$ of cases. Total thyroidectomy remains the only effective treatment, and only prior to metastasis; once metastatic disease has occurred, there is no effective therapy available. ${ }^{13}$

Although there is a well-established genotype-phenotype correlation for MEN2 patients, the mechanisms by which RET mutations cause tumors, the development of sporadic MTC in the absence of RET mutations, and the specific oncogenic pathways involved require further study. Our main aim was to identify signaling pathways specifically related to familial and sporadic MTC using transcriptional profiling.

\section{Materials and Methods}

\section{Human MTC Tissue Samples and Cell Lines}

Tumors $(n=68)$ from unrelated patients diagnosed with MTC were collected at the time of surgery from hospitals in Spain through the Spanish National Tumor Bank Network and from Italy through the Surgical Pathology Unit,
Department of Medical and Surgical Sciences, University of Padua. Tissue samples were frozen in liquid nitrogen, embedded in optimal cutting temperature compound (TissueTek OCT; Sakura Finetek, Torrance, CA), and stored at $-80^{\circ} \mathrm{C}$ until they were used for RNA extraction and hybridization onto arrays, or for RET mutational screening (if germline genetic information on the patient was absent). All tissues were evaluated by pathologists using H\&E staining. Only samples with a high percentage $(>80 \%)$ of tumor cells were included in the study, and cases with high amyloid content were excluded. Additional independent FFPE MTCs $(n=23)$ were available to validate expression profiling through quantitative RT-PCR (RT-qPCR). After consent of each patient was obtained, clinical information was collected by questionnaire. Genetic and general characteristics of the cases are listed in Supplemental Table S1.

Two tissue microarrays (TMAs) were available for further analysis (Supplemental Table S2). The first, TMA-1, contained 54 independent molecularly characterized FFPE MTC tissues; of these, 12 carried a germline RET mutation in codon 634, 9 had a somatic M918T mutation, and 22 were classified as WT because no mutations were found. [Other MTCs were not included in the analysis as they carried different mutations (3 MTCs with the C618F mutation, 1 MTC with C618R, 1 MTC with S891A, 1 MTC with C620F, 1 MTC with A883F), or the cores included in the TMA were not useful for immunostaining evaluation (2 negative MTC and 2 with mutation at 634 residue). These four tumors are referred as to "not used" in Supplemental Table 2. This TMA also contained four other thyroid cancer types and two normal tissues used as controls, as well as normal thyroid tissue of 13 MTC cases included in the TMA. TMA-2 was constructed with cores from the corresponding paraffin-embedded material from 13 frozen samples used for profiling purposes, along with two MTCs that were not hybridized, two thyroid cancers of different subtype, and 12 normal thyroid tissues from the corresponding MTCs. This TMA contained MTCs from patients carrying a germline RET mutation in codon 634 and somatic RET mutations affecting codon 918, as well as MTCs from patients classified as WT.

MZ-CRC-1 cells are derived from a metastatic MTC and harbor the $R E T^{M 918 T}$ mutation. ${ }^{14,15}$ The TT cell line has a codon 634 mutation in the RET gene.

\section{Molecular Characterization of Tumors}

Screening of RET was performed by direct sequencing of exons 10,11 , and 13 to 16 in DNA extracted from peripheral blood samples (when available) and, if negative, from tumor specimens. Genomic tumor DNA was obtained according to the manufacturer's instructions using a DNeasy kit (Qiagen, Valencia, CA).

\section{RNA Isolation}

Total RNA from 68 frozen MTC tissues was obtained according to the manufacturer's instructions using a TRI 
Reagent kit (MRC, Cincinnati, OH). RNA purity and integrity was assessed using an Agilent 2100 bioanalyzer (Agilent Technologies, Santa Clara, CA). RNA of 19 cases was degraded [RNA integrity number $(\mathrm{RIN})<7]$ and discarded for hybridization assays, but considered in the RT-qPCR validation step. Total RNA from 23 independent FFPE MTCs was isolated according to the manufacturer's instructions using an RNeasy FFPE kit (Qiagen). Concentration was determined using a NanoDrop ND-1000 spectrophotometer (Thermo Scientific, Wilmington, DE). These FFPE tumors were used for validation by RT-qPCR. After this first quality filter, 49 MTCs were used for hybridization assays and 42 (19 frozen and 23 FFPE) samples were used for validation purposes.

Total RNA from MZ-CRC-1 cells was extracted using an RNeasy kit (Qiagen).

\section{cDNA Synthesis, Labeling, Hybridization, and Detection}

For each of the 49 MTC tumors used for profiling, $500 \mathrm{ng}$ of total RNA was amplified according to the manufacturer's instructions by double-strand cDNA synthesis, followed by T7-based in vitro transcription. Universal Human Reference RNA (Stratagene, La Jolla, CA) was used as a control for all samples. Amplified complementary RNA (cRNA) was labeled with cyanine 5-conjugated dUTP, whereas cRNA from Universal Human Reference RNA was labeled with cyanine 3-conjugated dUTP. The Agilent Whole Human Genome Array $(4 \times 44 K)$ was used for competitive hybridization, and the slides were washed, dried, and scanned in an Agilent microarray scanner (Agilent Technologies).

\section{Normalization and Preprocessing}

Two-channel arrays were hybridized and quantified using Feature Extraction software version 9.5 (Agilent Technologies). Microarray background substraction was performed using the normexp method. ${ }^{16}$ The data set was normalized using loess within-array normalization and quantile between-array normalization. Differentially expressed genes were identified by applying linear models using the Bioconductor limma R package. ${ }^{17}$ Normalized data were preprocessed using the Gene Expression Profile Analysis Suite (GEPAS) server. ${ }^{18}$ Inconsistent replicates (SD > 1) were discarded, and consistent replicates were averaged. Finally, genes that exhibited flat patterns $(\mathrm{SD} \geq 0.8)$ across the set of samples were filtered and omitted in further comparisons.

\section{Unsupervised and Supervised Analysis}

Tumor samples were grouped according to their expression profiles by means of unsupervised clustering using GeneCluster 2.0. ${ }^{19}$ Unsupervised clustering was implemented by a self-organizing maps (SOM) algorithm. Only tumors exhibiting the 634 or the M918T mutation, along with tumors classified as WT, were used in further analysis.
Gene Expression Profiling and Gene Ontology Analysis

The Pomelo II toolset was used for $t$-testing, ${ }^{20}$ and the T-Rex toolset from the GEPAS package was used for differential expression analysis. ${ }^{18}$ To account for multiple hypothesis testing, the estimated significance level ( $P$ value) was adjusted using Benjamini's false discovery rate (FDR) correction. ${ }^{21}$ Genes with FDR $<0.05$ and with fold change $>2$ were selected for the validation step.

Functional enrichment of gene ontologies (GOs) for differentially expressed genes was performed using the FatiGO tool ${ }^{22}$ implemented in Babelomics software version 3.2 (Institute of Computational Genomics-CIPF, Valencia, Spain). Fisher's exact test was performed to obtain significant over-representation of GO terms in the list of differentially expressed genes versus the rest of the genome. GO terms with FDR $<0.05$ were considered significant.

Gene Set Enrichment Analysis (GSEA) ${ }^{23}$ using Biocarta and KEGG annotations was applied. Gene expression values were ranked based on the $t$-statistic. Gene sets with FDR $<0.25$ were considered enriched between classes under comparison.

\section{Validation by RT-qPCR}

For validation of microarray data, five genes significantly differentially expressed among profiling results were selected to be assessed by RT-qPCR: LOXL2, PROM1, GAL, GFRA1, and DKK4.

Out of 49 previously hybridized samples, 32 MTCs were used for validation, along with 42 available MTCs from an independent series (19 frozen MTC tissues not used in profiling and 23 FFPE tumors). After testing $\beta$-glucuronidase (GUS), 60S acidic ribosomal protein $\mathrm{P} 0$ (RPLPO), and peptidylprolyl isomerase A (PPIA) genes as endogenous references, only $G U S$ was used to normalize the quantification of mRNA expression.

Reverse transcription was performed using $1 \mu \mathrm{g}$ total RNA and random hexamers (FFPE samples) or $0.5 \mu \mathrm{g}$ total RNA and oligo-dT (frozen specimens) and M-MLV Reverse Transcriptase (Promega, Madison, WI). PCR was performed on an ABI Prism 7900 sequence detection system (Applied Biosystems; Life Technologies, Foster City, CA) using the Universal Probe Library set according to the manufacturer's recommendations. PCR reactions were performed in triplicate. The KruskalWallis test (nonparametric analysis of variance) for multiple class comparisons and the nonparametric $U$-test for two-class comparisons were used to compare the genetic classes.

For MZ-CRC-1 cells, total RNA was reverse-transcribed using a TaqMan reverse transcription kit (Applied Biosystems; Life Technologies, Foster City, CA). The relative mRNA levels were measured by RT-qPCR using TaqMan probes (Hs01009250_m1 for PROM1 and Hs99999905_m1 for GAPDH) (Life Technologies). GAPDH was used for data normalization. Quantification data were calculated by the $2^{-\Delta \Delta C T}$ method. 


\section{Cell Culture and Nucleofection}

MZ-CRC-1 and TT cells were maintained in Dulbecco's modified Eagle's medium supplemented with $10 \%$ heatinactivated fetal bovine serum, $1 \%$ pyruvate, $1 \%$ nonessential amino acids, $100 \mathrm{U} / \mathrm{mL}$ penicillin and streptomycin at $37^{\circ} \mathrm{C}$ in $5 \% \mathrm{CO}_{2}$ in air. For nucleofection, $2 \times 10^{6}$ cells per condition were incubated with $2 \mu \mathrm{mol} / \mathrm{L}$ synthetic siRNAs (Sigma-Aldrich, St. Louis, MO) directed against human PROM1 in a solution containing $100 \mathrm{mmol} / \mathrm{L}$ phosphate buffer, $100 \mathrm{mmol} / \mathrm{L} \mathrm{KCl}$, and $10 \mathrm{mmol} / \mathrm{L} \mathrm{MgCl}_{2}$. Cells were nucleofected using the A30 program of an Amaxa Nucleofector II device (Lonza, Walkersville, MD). Immediately after nucleofection, cells were resuspended in medium and seeded onto six-well plates.

\section{Apoptosis Assay}

Three days after transfection, the number of apoptotic nuclei was analyzed by Hoechst staining as described previously. ${ }^{24}$ Cell-permeant Hoechst dye 33342 was directly added to cells at a final concentration of $0.5 \mathrm{mg} / \mathrm{mL}$ and incubation was continued for 15 minutes at $37^{\circ} \mathrm{C}$. Cells were then photographed under a fluorescence microscope (IX70; Olympus, Tokyo, Japan). Cells with condensed and/or fragmented chromatin typical of apoptosis were scored in triplicate as percentage of total nuclei. For each condition, 500 to 1000 cells were counted.

\section{Lentivirus Construction and Infection}

An expression cassette consisting of the cytomegalovirus (CMV) promoter followed by the human Prominin 1 open reading frame was cloned into a modified pDSL_hpUG vector (10326371; ATCC, Manassas, VA) lacking the ubiquitin c-GFP cassette. Briefly, the Prominin 1 open reading frame was first cloned into pcDNA3 (Invitrogen; Life Technologies, Carlsbad, CA) by standard techniques. Using such construct as a template, a PCR product consisting of the CMV promoter followed by the Prominin 1 open reading frame and flanked by attB sites was generated. Finally, this product was sequentially cloned into pDONR221 entry vector (Invitrogen; Life Technologies) and then moved to the final destination vector via gateway recombination technology.

Lentiviral particles were produced as follows. Briefly, 293T cells were cotransfected by the calcium phosphate method with the above plasmid plus plasmids coding for the envelope and the packaging systems (VSV-G and $\Delta 8.9$, respectively). The day after transfection, the culture medium was replaced with fresh medium and left for an additional 2 to 3 days to allow lentiviruses to be produced and released. Supernatants were then harvested, filtered through a $0.45-$ $\mu \mathrm{m}$ filter, and directly applied to TT cells. Typically, cells were infected overnight and experiments were performed 4 to 5 days after infection.

\section{RT-qPCR}

Total RNA from MZ-CRC-1 cells was extracted with an RNeasy kit (Qiagen) and reverse-transcribed using a TaqMan Reverse Transcription Kit (Applied Biosystems; Life Technologies). The relative mRNA levels of CD133 were measured by RT-qPCR using TaqMan probes (Hs01009250_m1 for CD133 and Hs99999905_m1 for GAPDH (Life Technologies). GAPDH was used for data normalization. Quantification data were calculated by the $2^{-\Delta \Delta C T}$ method.

\section{BrdU Incorporation}

Cells were incubated with $3 \mathrm{ng} / \mathrm{mL} 5^{\prime}$-bromodeoxyuridine (BrdU; Sigma-Aldrich) for 20 minutes and fixed with $4 \%$ paraformaldehyde. After DNA denaturing with $2 \mathrm{~mol} / \mathrm{L} \mathrm{HCl}$ for 30 minutes and neutralization with $0.1 \mathrm{~mol} / \mathrm{L} \mathrm{Na}_{2} \mathrm{~B}_{4} \mathrm{O}_{7}$ (pH 8.5) for 2 minutes, cells were blocked in PBS solution containing 5\% horse serum, $5 \%$ fetal bovine serum, $0.2 \%$ glycine, and $0.1 \%$ Triton X-100 for 1 hour. Subsequently, cells were subjected to indirect immunofluorescence with a mouse anti-BrdU monoclonal antibody (Dako, Carpinteria, CA) and Alexa Fluor 594-conjugated anti-mouse secondary antibody (Invitrogen; Life Technologies). Nuclei were counterstained with $5 \mu \mathrm{g} / \mathrm{mL}$ Hoechst dye 33258, and cells were visualized using an epifluorescence microscope (Leica Microsystems, Wetzlar, Germany).

\section{Cell Viability}

Cell viability was determined by the 3-(4,5-dimethylthiazol2-yl)-2,5'-diphenyltetrazolium bromide (MTT) assay. TT and MZ-CRC-Z cells were plated on 96-well dishes at $2 \times 10^{4}$ cells per well. Next day, cells were treated with the Ret inhibitor RPI-1, which has been shown to induce apoptosis in MTC-derived cell lines. ${ }^{25,26}$ After 48 hours of treatment, cells were incubated for 35 to 45 minutes with 0.5 $\mathrm{mg} / \mathrm{mL}$ of MTT reagent (Sigma-Aldrich), washed, and solubilized with dimethyl sulfoxide. Absorbance was measured at $595 \mathrm{~nm}$ in a microplate reader (Bio-Rad Laboratories, Hercules, CA).

\section{Immunohistochemistry}

Immunohistochemical staining was performed on both of the MTC tissue microarrays available. TMA blocks were sectioned at a thickness of $3 \mu \mathrm{m}$ and dried for 1 hour at $60^{\circ} \mathrm{C}$. Deparaffinization, rehydration, and epitope retrieval were achieved by heat treatment in a PT Link module (Dako, Glostrup, Denmark) at $\mathrm{pH}$ 9. Before staining of the sections, endogenous peroxidase was blocked. Primary antibodies used were anti-PROM1 (1:500, ab19898; Abcam, Cambridge, MA) and anti-GFRA1 (1:500, C-20; Santa Cruz Biotechnology, Santa Cruz, CA); incubation with primary antibodies was performed according to the manufacturer's instructions. After incubation with anti-PROM1, the reaction 
was visualized with EnVision FLEX system (Dako) using diaminobenzidine chromogen as a substrate; anti-GFRA1 binding was visualized with an LSAB + kit (Dako) using diaminobenzidine chromogen as a substrate. Sections were counterstained with hematoxylin. Appropriate positive and negative controls were also tested.

Immunoexpression was graded semiquantitatively by considering the percentage and intensity of the staining. A histological score was obtained for each sample, on a scale from 0 (no immunoreaction) to 300 (maximum immunoreactivity). Interpretation was performed as described previously. ${ }^{27}$ Because each TMA included two cores from each case, immunohistochemical evaluation was performed after examination of all samples.

\section{Statistical Analysis}

Immunoexpression was analyzed in two ways, as a continuous variable and as a binary variable using the median as the cutoff. For the first analysis, a $U$-test was used; for the second analysis, difference in expression was tested with a $\chi^{2}$ test or Fisher's exact test, as appropriate. Statistical analyses were performed using SPSS software package version 17.0 (IBM SPSS, Chicago, IL). Nominal two-sided $P$ values of $<0.05$ were considered statistically significant.

\section{Results}

\section{Molecular Genetic Characteristics of the MTC Tumors}

Tumors were classified according to their specific RET mutation. Among the 49 tumors used for profiling, 7 carried a germline RET mutation in codon 634, 14 carried the M918T RET mutation (1 germline and 13 somatic mutations), and 20 were classified as WT. The remaining eight MTCs had six different germline mutations and two different somatic mutations in RET (Supplemental Table S1). These last eight tumors were not considered in clustering analysis.

\section{Data Reveal Differential Expression Profiles in Inherited and Sporadic MTC}

Unsupervised hierarchical clustering, performed using standard correlation as a measure of similarity across all hybridized samples, showed a relatively low heterogeneity distributed over two principal branches (Figure 1). An enrichment of familial MTC cases with various germline RET mutations was observed in the second branch of the cluster, whereas the first branch was enriched for sporadic MTC with somatic $R E T^{M 918 T}$ mutation and for WT cases. This observation led us to do a supervised analysis based on the known mutations of each available tumor. Differential expression analysis was performed to compare $\mathrm{MTC}^{\mathrm{M} 918 \mathrm{~T}}$ versus MTC $^{\mathrm{WT}}$ and $\mathrm{MTC}^{\mathrm{M} 918 \mathrm{~T}}$ versus MTC ${ }^{634}$ (Figures 2 and 3), from which we obtained a list of 360 and 18 significant genes (GEO accession number GSE32662), respectively.
Based on threshold-free methodology using Gene Set Enrichment Analysis software version 2.08 (GSEA; Broad Institute, Cambridge, MA), signaling pathways were identified as significantly differently expressed (FDR $<25 \%$ ) for comparison of MTC $^{\text {M918T }}$ versus MTC $^{634}$ and MTC $^{\mathrm{M} 918 \mathrm{~T}}$ versus MTC ${ }^{\mathrm{WT}}$ (Supplemental Table S3). The M918T cluster was correlated with several pathways related to malignant tumor behavior, such as the p53, caspase, cytokine, Wnt, NFKB, MAPK, IL1R, JAK/Stat, and Notch pathways.

Gene Ontology (GO) analyses revealed significant biological processes in the M918T group associated with malignant behavior (Supplemental Table S4). Hierarchical clustering analysis of the most relevant FatiGO outputs for biological processes pointed to the importance of cell adhesion, migration, and proliferation ontologies (data not shown).

GSEA analysis (Supplemental Table S3) and FatiGO analysis (Supplemental Table S5) revealed similar significant pathways associated with the M918T group.

\section{Biomarkers of Specific Genetic Conditions}

After application of the filtering criteria, five genes appeared to be biomarkers of different genetic conditions: PROMI, GFRA1, LOXL2, GAL, and DKK4. These were selected for further validation at the mRNA level.

$G A L$ was the only statistically significant gene found to be a specific marker for the germline RET mutation in codon 634. The expression of the other four genes was validated by RT-qPCR as up-regulated markers in $\mathrm{MTC}^{\mathrm{M} 918 \mathrm{~T}}$. Because of limited availability of RNA, it was possible to validate PROM1 and LOXL2 only in FFPE MTC samples. These results are summarized in Figure 4 for the independent frozen series and in Figure 5 for the FFPE series.

Although they were not selected for further validation, other genes differentially expressed among the three different genetic conditions considered here have been reported to be involved in cancer of tissues derived from the same neural crest origin as MTC, and also in the RTK signaling pathway. These previous findings were therefore considered to be a cross-validation of our results. Among these markers, the up-regulated genes associated with the M918T group were ESM1, NPR1, PITX2, RTN1, and SHC1.

ESM1 (encoding endothelial cell-specific molecule 1) is associated with cell migration and proliferation; its overexpression has been described previously in sporadic $R E T^{M 918 T}$ MTC cases. ${ }^{28}$ NPRI (encoding atrial natriuretic peptide receptor $1 /$ guanylate cyclase A) regulates the activation of ERK1/2, ${ }^{29}$ as well as the Ras/Raf/ERK and Wnt/ $\beta$-catenin pathways. PITX2 (encoding paired-like homeodomain transcription factor 2 ) is involved in tumorigenesis and in $\mathrm{Wnt} / \beta$-catenin signaling; it is expressed in neural crest cells. ${ }^{30}$ RTN1 (encoding reticulon-1), overexpression of which leads to apoptosis of neuroblastoma cell lines, is involved in secretion or membrane trafficking in neuroendocrine cells as well as in tumorigenesis; it is 


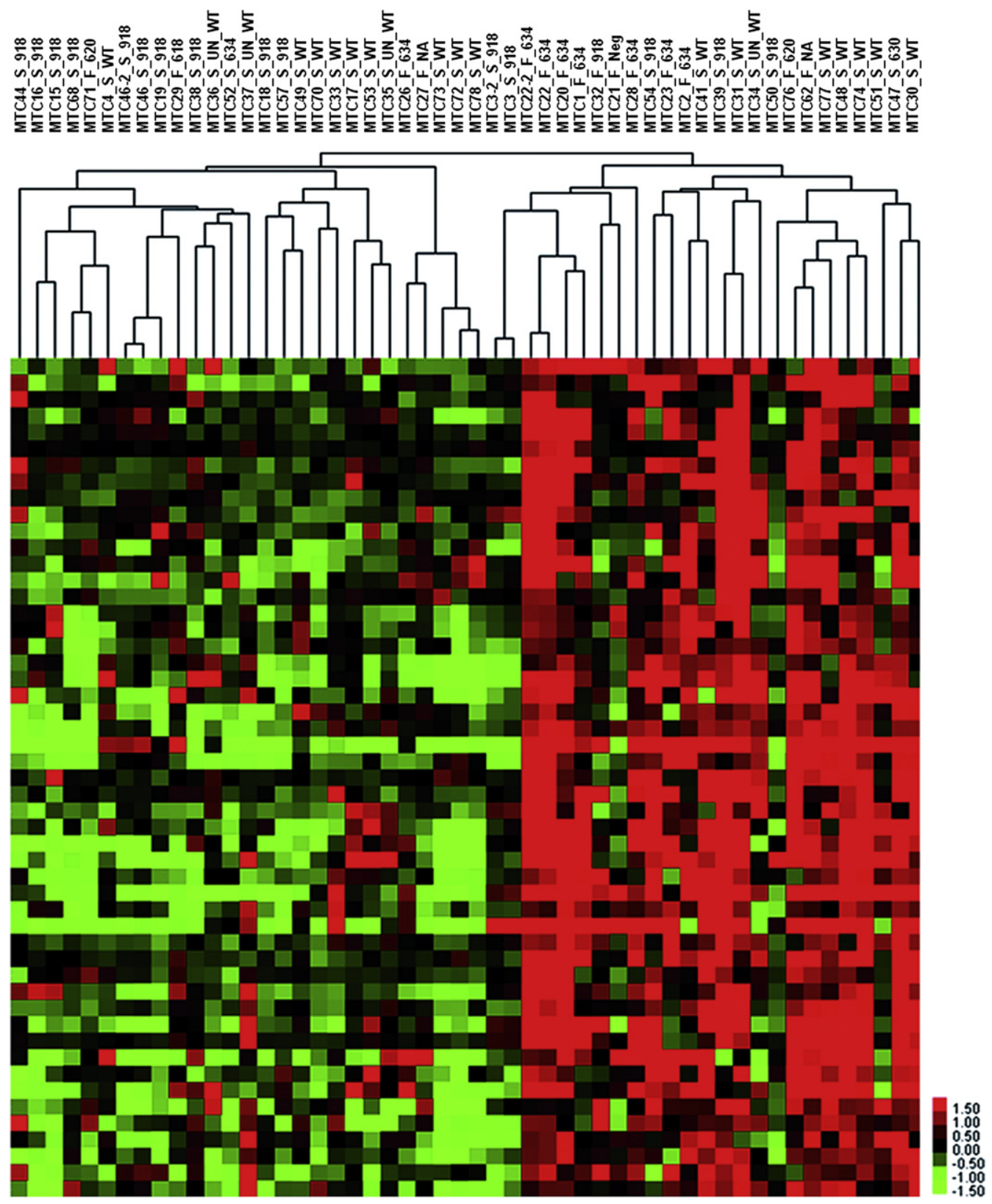

Figure 1 Unsupervised clustering of hybridized frozen MTC series. Partial heat map shows two principal branches after unsupervised clustering was applied to the 49 MTCs. The first branch was enriched with MTC ${ }^{\text {M918T }}$ cases and the second with familial MTC cases, mostly with RET $T^{634}$ mutation. Relative underexpression is shown as shades of green and relative overexpression is shown as shades of red. Cases are identified by MTC case number and a mutation extension: S_918 for sporadic MTC with somatic RET ${ }^{\text {M918T }}$ mutation, F_634 for familial MTC with RET $T^{634}$ mutation, and S_WT for sporadic MTC with no RET mutation.

expressed in neurons and neuroendocrine cells. ${ }^{31}$ Fibroblasts transformed by $R E T^{C 634 R}$ display constitutive RETmediated phosphorylation of SHC, which activates the RAS/MAPK signaling pathway. ${ }^{32}$

\section{Immunohistochemistry}

We performed immunohistochemistry for GFRA1 and PROM1 (Supplemental Figure S1). Results were evaluated according to uniform pre-established criteria.

Differences at the mRNA level were not observed at the protein level. GFRA1 protein levels were not significantly different among the groups. PROM1 showed higher staining in $\mathrm{MTC}^{\mathrm{M} 918 \mathrm{~T}}$ than in $\mathrm{MTC}^{634}(P=0.059)$, in accord with mRNA detection.

\section{Silencing PROM1 by siRNA Induces Apoptosis}

We used the MZ-CRC-1 cell line nucleofected with two different synthetic siRNAs against human PROM1 for in vitro validation. Knockdown efficiency was assessed in parallel dishes by RT-qPCR, and the number of apoptotic nuclei was scored as percentage of total nuclei. Both siRNAs caused an increase in the number of apoptotic 


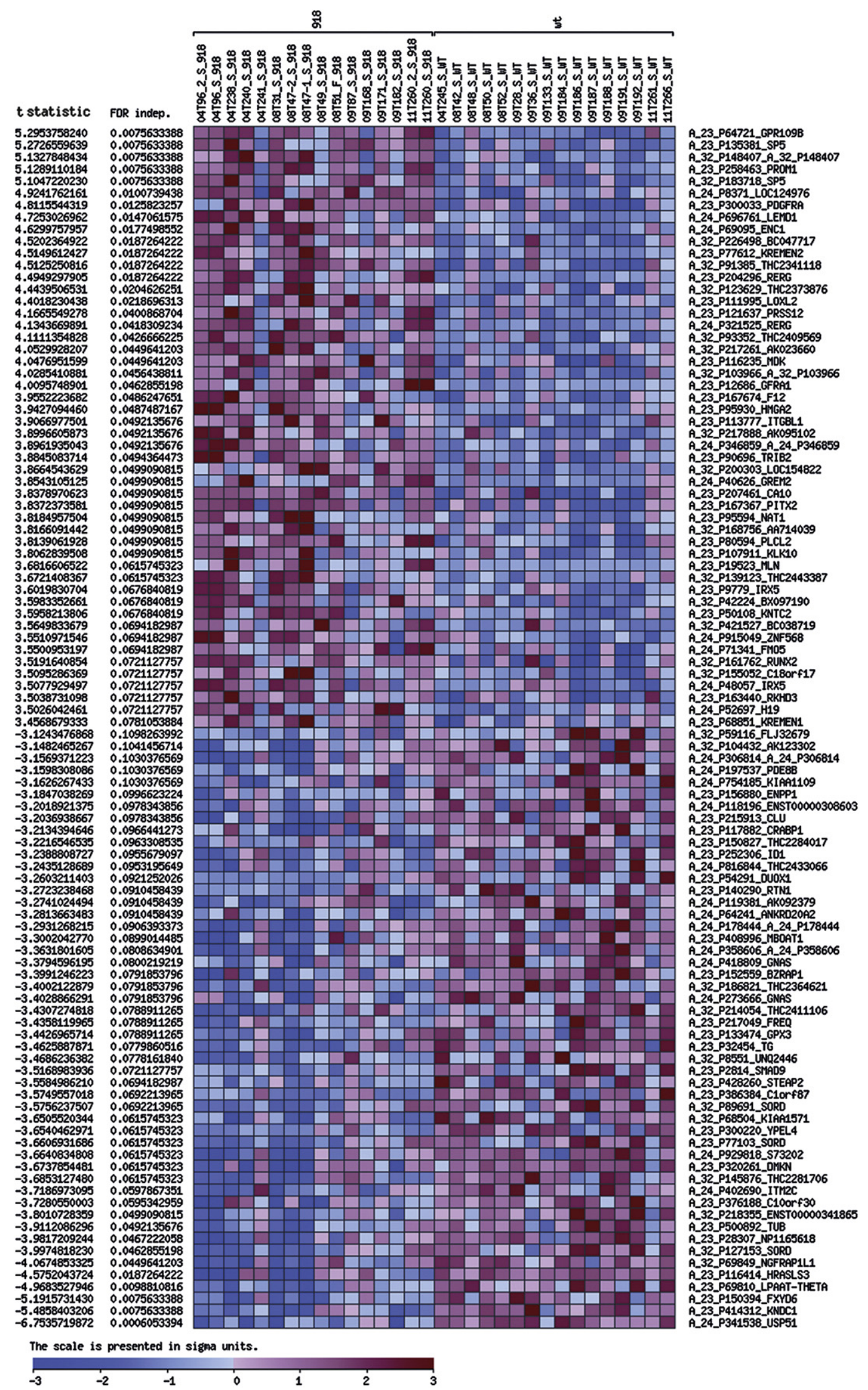

Figure 2 Differential expression analysis of MTC ${ }^{\text {M918T }}$ versus MTC ${ }^{\text {WT }}$ using the GEPAS package. The first 100 genes with FDR $<0.15$ are shown. Genes are listed in descending order by $t$-statistic. Sigma units denote standard deviation. 


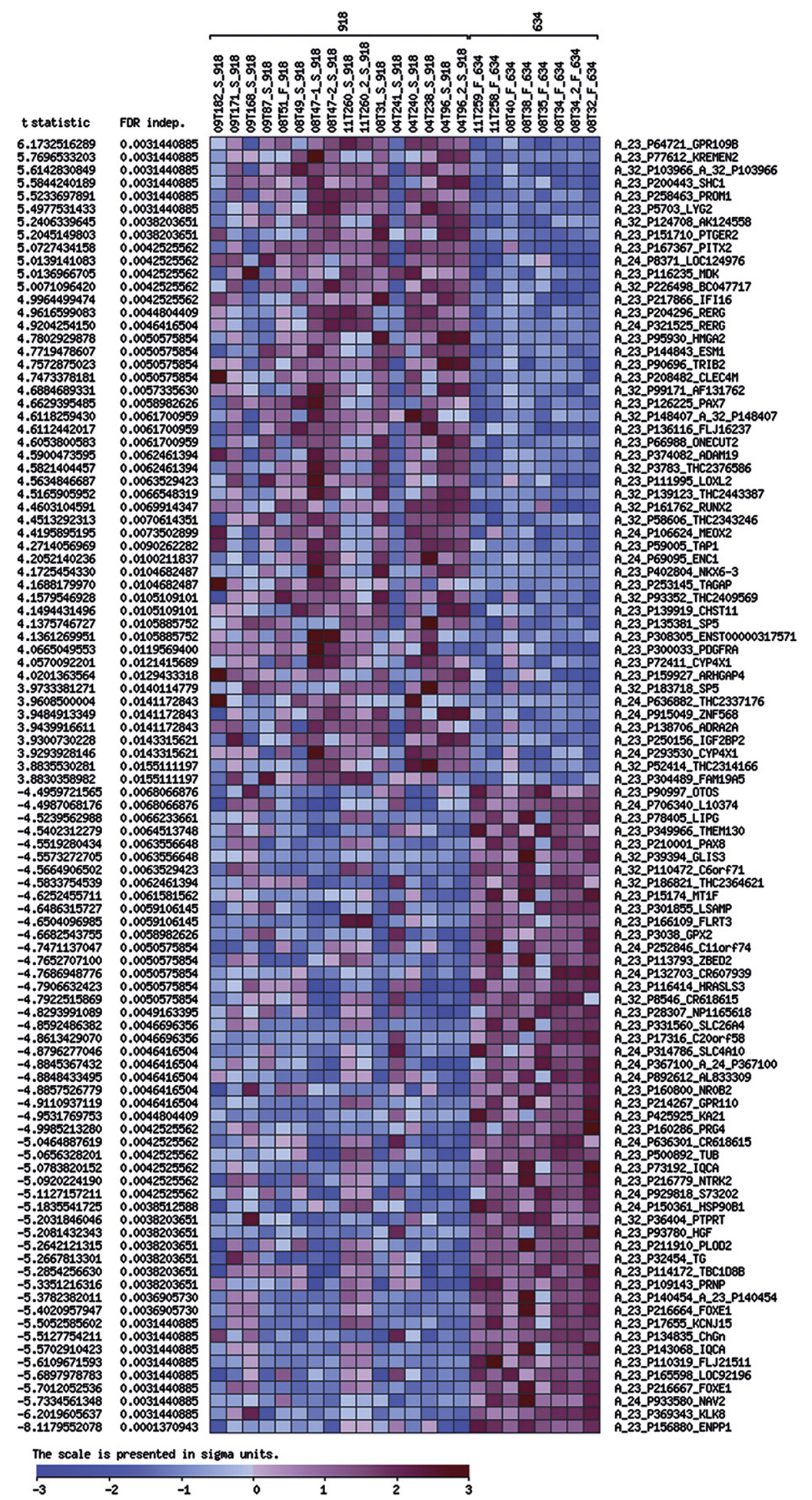

Figure 3 Differential expression analysis of MTC ${ }^{\text {M918T }}$ versus MTC $^{634}$ using the GEPAS package. The first 100 genes with FDR $<0.05$ are shown. Genes are listed in descending order by $t$-statistic. 


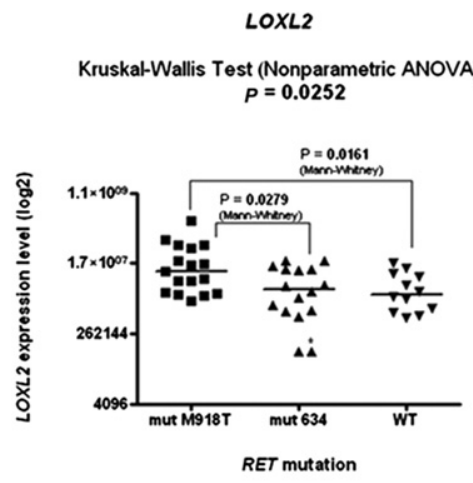

GAL

Kruskal-Wallis Test (Nonparametric ANOVA) $P=0.0002$

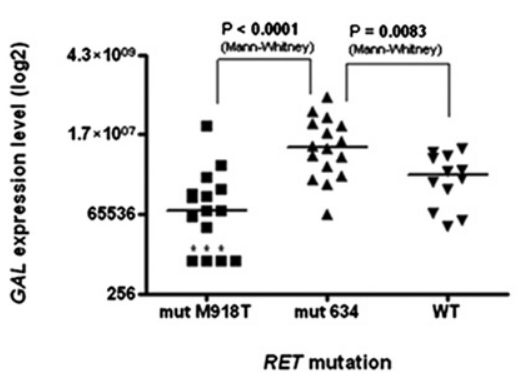

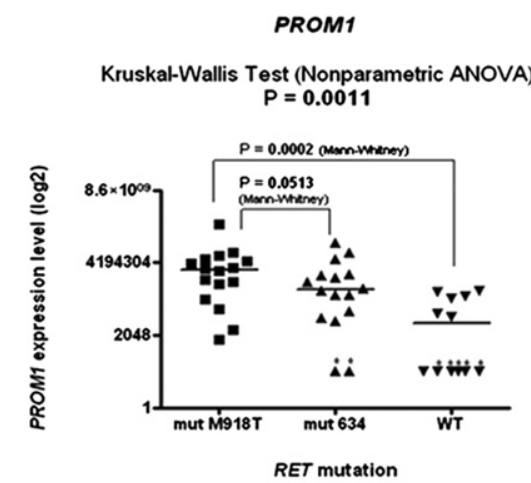

GFRA1

Kruskal-Wallis Test (Nonparametric ANOVA) $P=0.0576$

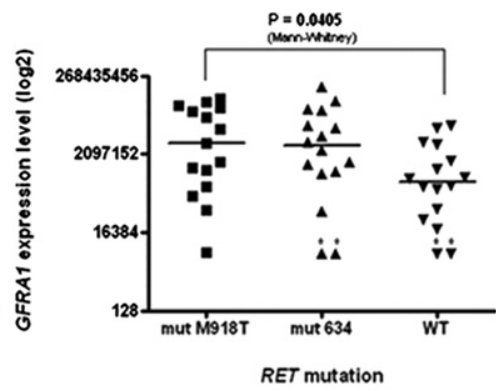

DKK4

Kruskal-Wallis Test (Nonparametric ANOVA) $P=0.0014$

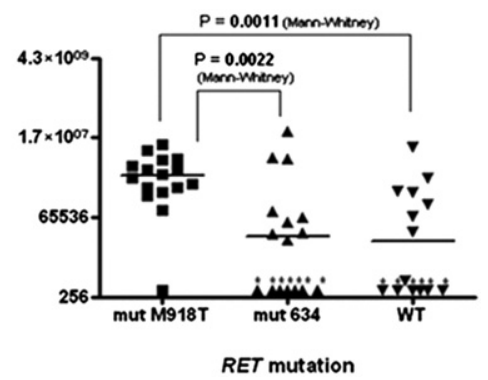

Figure 4 RT-qPCR with TaqMan probes of LOXL2, PROM1, GAL, GFRA1, and DKK4 expression in an independent frozen MTC validation series. Asterisks indicate values below the lowest detected $C_{T}$ value. ANOVA, analysis of variance.

nuclei (Figure 6), suggesting that PROM1 is necessary for the survival of these cells. We next analyzed PROM1 expression levels in two MTC-derived cell lines bearing a MEN2B RET mutation (MZ-CRC), and a MEN2A RET mutation (TT). In accord with our results in MTC specimens, MZ-CRC expressed PROM1 mRNA levels approximately fivefold higher than those found in TT cells. To check whether overexpression of PROM1 in TT cells could confer a growth advantage to TT cells, we overexpressed PROM1 in these cells by means of lentiviral infection. Overexpression of PROM1 did not affect the proliferation rate of TT cells as measured by BrdU uptake, but did afford protection against cell death caused by the RET inhibitor RPI- ${ }^{25}$ (Figure 6). Expression of PROM1 thus appears to influence cell death, rather than proliferation.

\section{Discussion}

Survival of MTC patients is relatively low if their carcinomas metastasize ${ }^{33}$ Surgery is the primary treatment for MTC, and alternatives are lacking for tumors with poor response to either conventional chemotherapy or radiotherapy. ${ }^{33}$ Thus,
LOXL2

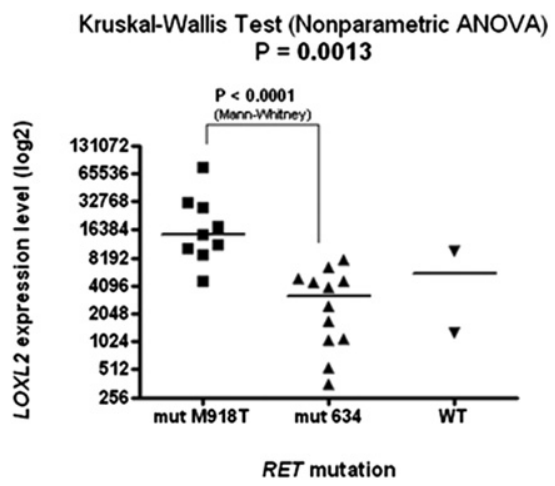

PROM1

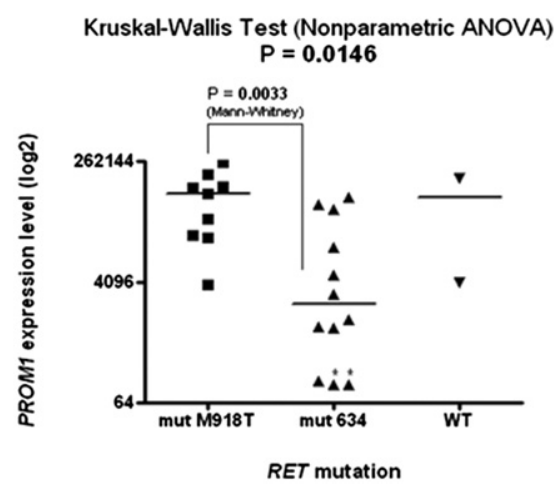

Figure 5 RT-qPCR with TaqMan probes of LOXL2 and PROM1 expression in an independent FFPE MTC validation series. Asterisks indicate values below the lowest detected $C_{T}$ value. 
A
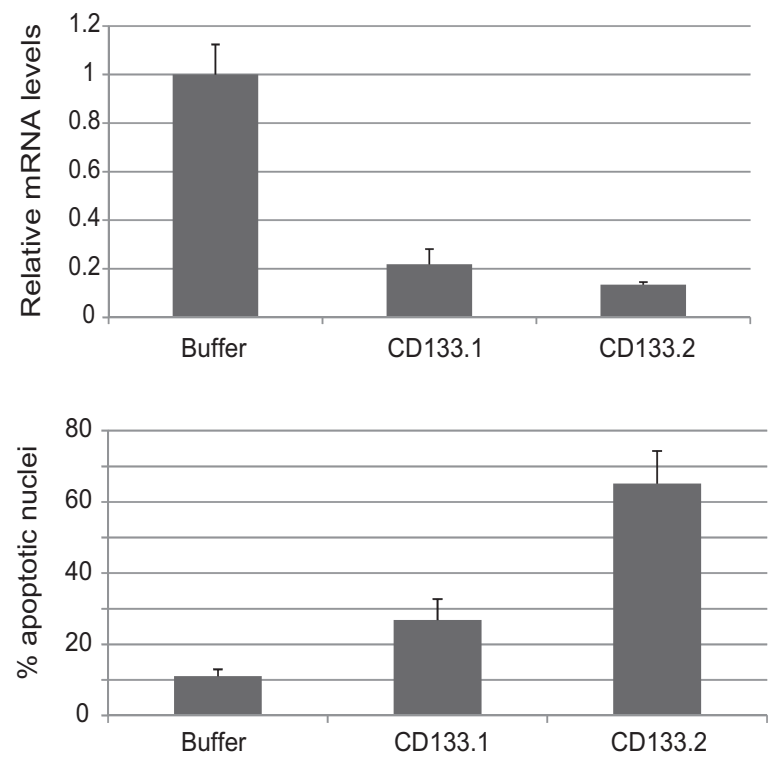

B

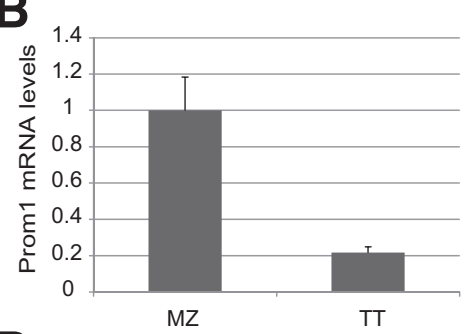

D

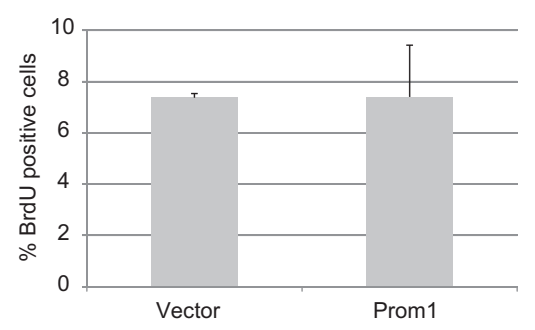

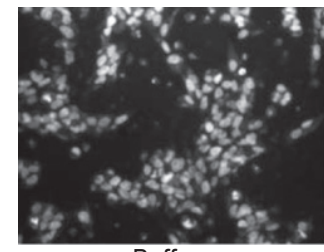

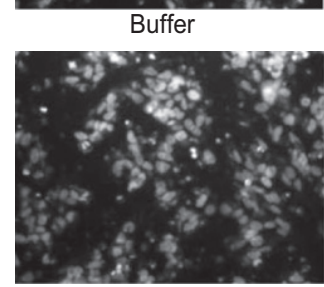

CD133.1

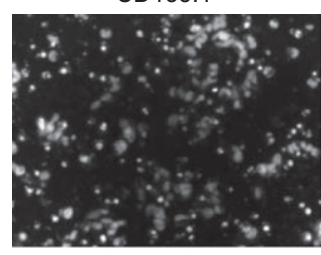

CD133.2

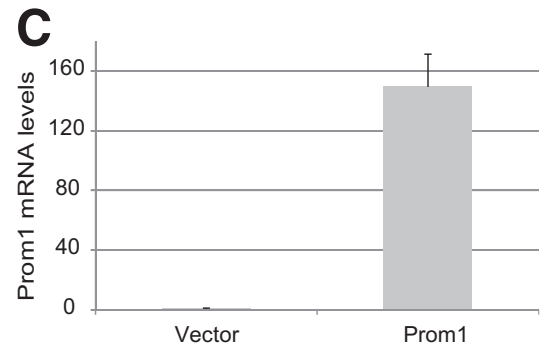

E

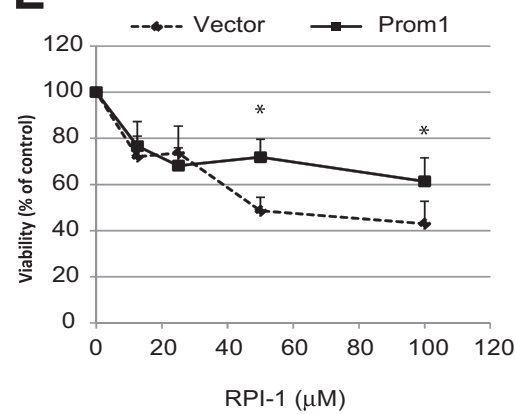

Figure 6 A: Knockdown efficacy of two different siRNAs against human PROM1. MZ-CRC-1 cells were nucleofected with either buffer or siRNAs against PROM1 (CD133.1 and CD133.2). Three days later, PROM1 expression was assessed by RTqPCR (top left). Silencing of PROM1 causes apoptotic death of MZ-CRC-1 cells. Cells were nucleofected as above; 3 days later, the number of nuclei showing apoptotic morphology was scored (bottom left). Representative micrographs of Hoechst 33342-stained nuclei of cells (right). Original magnification, $\times 100$. B: mRNA levels of PROM1 of MZ-CRC-1 and TT cell lines. C: mRNA levels of PROM1 after lentiviral infection with empty vector or PROM1. D: BrdU uptake of TT cells transduced with lentivirus. E: Cell viability of TTderived cell lines after incubation with the RET inhibitor RPI-1. ${ }^{*} P<0.01$ two-tailed Student's $t$ test. there is a pressing need to identify potential therapeutic targets. Although a genotype-phenotype correlation has been established for MEN2 cases, and it is known that clinical behavior differs according to specific germline and somatic RET mutations, the signaling pathways involved in the clinical behavior are largely unknown. It is therefore important to identify pathways related to non-RET-dependent sporadic MTC and to the most frequent pathogenic RET mutations, to reveal prognostic markers. Although this need is well recognized, several difficulties have contributed to a lack of studies on MTC tumors. First, comparisons of primary tumors versus normal $\mathrm{C}$ cells are not possible, because of the very low parafollicular cell representation $(\sim 1 \%)$ in the thyroid cell mass. ${ }^{34}$ In addition to these difficulties, it is a challenge to obtain a sufficiently large series of frozen MTC tissues for genomic study, because of the rarity of MTC. This explains why so few studies on MTC profiling have been reported. ${ }^{28,35-38}$ The present study, based on one of the largest series of primary MTCs described to date, was focused on finding differences among expression profiles according to the genetic condition of the MTC samples.

\section{Biomarkers and Pathways Are Specific to Each Genetic Class of MTCs}

In accord with our finding in $\mathrm{MTC}^{634}, G A L$ overexpression has been observed in PC12 rat pheochromocytoma cell lines. $^{39}$ GAL is involved in neuropeptide signaling, 
tumorigenesis, nervous system development, and the regulation of cell proliferation; it is expressed in neuroblastoma and pheochromocytoma cancer cell lines, and it activates MAPK and ERK signaling. ${ }^{39-42}$ The present findings suggest that $G A L$ overexpression may be involved in the development of the phenotype exhibited by MEN2A patients carrying a germline $R E T^{634}$ mutation; pheochromocytoma develops in at least $50 \%$ of these cases. ${ }^{3,43}$

GDNF family receptor $\alpha-1$ (encoded by GFRA1), associated with MTC $^{\mathrm{M} 918 \mathrm{~T}}$, is a known coreceptor of RET that mediates its activation through the binding of RET ligands. These include ligands that are widely expressed and promote the survival of neurons during development. GFRAl has been previously reported as a genetic risk factor associated with susceptibility to developing sporadic MTC in a case-control study, ${ }^{44}$ and our results support its role in development of MTC.

DKK4 (encoding Dickkopf-related protein 4), another gene overexpressed in $\mathrm{MTC}^{\mathrm{M} 918 \mathrm{~T}}$, is significantly up-regulated in colorectal cancer, and this up-regulation reflects activation of the Wnt canonical pathway. ${ }^{45}$ Furthermore, KREMEN2 (encoding kringle containing transmembrane protein 2) cooperates with $D K K 4$ to regulate Wnt signaling. ${ }^{46} K R E$ $M E N 2$ was up-regulated similar to $D K K 4$ in M918T tumors included in the present study. Deregulation of the Wnt pathway results in the development of cancer and other diseases, and Wnt/ $\beta$-catenin signaling and JNK noncanonical pathways are implicated in RET signaling, which is activated in MTC. Deregulation of the Wnt pathway specifically associated with $R E T^{M 918 T}$ MTC could open new perspectives for treatment.

The most noteworthy results among the M918T group were the overexpression of LOXL2 (GEO accession number GSE32662) and PROM1. LOXL2 (encoding lysyl oxidaselike protein 2) activates Snail/E-cadherin and Src kinase/ focal adhesion kinase (Src/FAK) pathways and is associated with tumor cell invasion. LOXL2 is also linked to epithelialmesenchymal transition, which leads to metastasis and lower survival rates in epithelial and neuroendocrine tumors. ${ }^{47-50}$ Increased expression of genes associated with epithelial-mesenchymal transition has been previously observed in MEN2B MTCs. ${ }^{38}$

PROM1 was the gene most significantly overexpressed among the M918T MTCs included in our series of primary tumors. PROMI was recently reported by $\mathrm{Zhu}$ et al ${ }^{51}$ to be a cancer stem cell marker in MZ-CRC-1 cell lines harboring a $R E T^{M 918 T}$ mutation. Because MTCs bearing the $R E T^{M 918 T}$ mutation are resistant to treatment, a high expression level of PROM1 might be related to resistance to therapy. In this regard, high expression of $P R O M 1$ is associated with neural crest-derived stem cells, and slowly differentiating cancer stem cells have been proven to be resistant to irradiation and cytotoxic drugs. ${ }^{51}$ To our knowledge, the present study is the first study based on primary tumors that points to PROM1 overexpression associated with the M918T mutation. Another study recently showed an association of poor prognosis with a high expression of PROMI in medulloblastoma tumors, suggesting that it might be a potential prognostic marker. ${ }^{52}$

Immunohistochemical assessment of GFRA1 and PROM1 in the 71 FFPE MTCs revealed a tendency toward association of the M918T group with PROM1 overexpression. Furthermore, after silencing of PROMI with two different siRNAs, we observed decreased levels of $P R O M 1$ mRNA which led to an increased number of apoptotic nuclei. This significant correlation between silencing of $P R O M I$ and apoptosis, taken together with our profiling data, led us to conclude that $P R O M I$ is a specific biomarker for the $R E T^{M 918 T}$ mutation and that its targeting seems feasible and could lead to apoptotic cell death. In this regard, GO biological function analyses using FatiGO and Babelomics revealed that important processes such as antiapoptotic functions, proliferation, migration, cell cycle, and adhesion were highly associated with the M918T group. Pathway enrichment analysis of the M918T group using GSEA software pointed to signaling cascades involved mainly in tumor invasion and metastasis $(\mathrm{NF} \kappa \mathrm{B}$, interleukin, cytokine, p38 MAPK, Wnt, JAK/Stat, Notch, MAPK, or cell adhesion), which agrees with what would be expected on the grounds of the clinical behavior of M918T-related MTCs. Among these pathways, the Notch pathway is known to play an important role in the normal development of neuroendocrine cells, including the calcitonin-producing $\mathrm{C}$ cells of the thyroid. $^{53}$

In summary, we have identified specific biomarkers related to different genetic conditions by means of expression analysis. The overexpression of LOXL2 (involved in the cell adhesion pathway and the epithelial-mesenchymal transition process) and of DKK4 (involved in the Wnt pathway), along with the fact that a decrease in PROMI expression was associated with an increased number of apoptotic nuclei, opens new therapeutic possibilities for improved management of MTC.

\section{Acknowledgment}

MZ-CRC-1 cells were a generous gift of Dr. Robert F. Gagel (MD Anderson Cancer Center, University of Texas, Houston, TX).

\section{Supplemental Data}

Supplemental material for this article can be found at http://dx.doi.org/10.1016/j.ajpath.2012.10.025.

\section{References}

1. Ball D, Baylin SB, De Butros AC: Medullary thyroid carcinoma. Werner and Ingbar's The thyroid, 8 ed. Edited by LE Braverman, RD Utiger. Philadelphia: Lippincott Williams \& Wilkins, 2000, pp 930-943

2. Pachnis V, Mankoo B, Costantini F: Expression of the c-ret protooncogene during mouse embryogenesis. Development 1993, 119: 1005-1017 
3. Eng C, Clayton D, Schuffenecker I, Lenoir G, Cote G, Gagel RF, van Amstel HKP, Lips CJM, Nishisho I, Takai SI, Marsh DJ, Robinson BG, Frank-Raue K, Raue F, Xue F, Noll WW, Romei C, Pacini F, Fink M, Niederle B, Zedenius J, Nordenskjold M, Komminoth P, Hendy GN, Gharib H, Thibodeau SN, Lacroix A, Frilling A, Ponder BAJ, Mulligan LM: The relationship between specific RET proto-oncogene mutations and disease phenotype in multiple endocrine neoplasia type 2. International RET mutation consortium analysis. JAMA 1996, 276:1575-1579

4. Mulligan LM, Kwok JB, Healey CS, Elsdon MJ, Eng C, Gardner E, Love DR, Mole SE, Moore JK, Papi L, Ponder MA, Telenius H, Tunnacliffe A, Ponder BA: Germ-line mutations of the RET protooncogene in multiple endocrine neoplasia type 2A. Nature 1993, 363:458-460

5. Mulligan L, Eng C, Healey CS, Clayton D, Kwok JB, Gardner E, Ponder MA, Frilling A, Jackson CE, Lehnert H, Neumann HPH, Thibodeau SN, Ponder BAJ: Specific mutations of the RET protooncogene are related to disease phenotype in MEN 2A and FMTC. Nat Genet 1994, 6:70-74

6. Machens A, Niccoli-Sire P, Hoegel J, Frank-Raue K, van Vroonhoven TJ, Roeher H-D, Wahl RA, Lamesch P, Raue F, ConteDevolx B, Dralle H; European Multiple Endocrine Neoplasia (EUROMEN) Study Group: Early malignant progression of hereditary medullary thyroid cancer. N Engl J Med 2003, 349:1517-1525

7. Dabir T, Hunter S, Russell C, McCall D, Morrison P: The RET mutation E768D confers a late-onset familial medullary thyroid carcinoma-only phenotype with incomplete penetrance: implications for screening and management of carrier status. Fam Cancer 2006, 5: 201-204

8. Hofstra RM, Fattoruso O, Quadro L, Wu Y, Libroia A, Verga U, Colantuoni V, Buys $\mathrm{CH}$ : A novel point mutation in the intracellular domain of the ret protooncogene in a family with medullary thyroid carcinoma. J Clin Endocrinol Metab 1997, 82:4176-4178

9. Drosten M, Pützer BM: Mechanisms of disease: cancer targeting and the impact of oncogenic RET for medullary thyroid carcinoma therapy. Nat Clin Pract Oncol 2006, 3:564-574

10. Kodama Y, Asai N, Kawai K, Jijiwa M, Murakumo Y, Ichihara M, Takahashi M: The RET proto-oncogene: a molecular therapeutic target in thyroid cancer. Cancer Sci 2005, 96:143-148

11. Marx SJ: Molecular genetics of multiple endocrine neoplasia types 1 and 2, [Erratum appeared in Nat Rev Cancer 2005, 5:663]. Nat Rev Cancer 2005, 5:367-375

12. Arighi E, Borrello MG, Sariola H: RET tyrosine kinase signaling in development and cancer. Cytokine Growth Factor Rev 2005, 16: 441-467

13. Weber T, Schilling T, Büchler MW: Thyroid carcinoma. Curr Opin Oncol 2006, 18:30-35

14. Cooley L, Elder FF, Knuth A, Gagel RF: Cytogenetic characterization of three human and three rat medullary thyroid carcinoma cell lines. Cancer Genet Cytogenet 1995, 80:138-149

15. Santoro M, Carlomagno F, Romano A, Bottaro DP, Dathan NA, Grieco M, Fusco A, Vecchio G, Matoskova B, Kraus MH, Di Fiore PP: Activation of RET as a dominant transforming gene by germline mutations of MEN2A and MEN2B. Science 1995, 267: 381-383

16. Ritchie M, Silver J, Oshlack A, Holmes M, Diyagamam D, Holloway A, Smyth GK: A comparison of background correction methods for two-colour microarrays. Bioinformatics 2007, 23: 2700-2707

17. Smyth GK, Michaud J, Scott HS: Use of within-array replicate spots for assessing differential expression in microarray experiments. Bioinformatics 2005, 21:2067-2075

18. Montaner D, Tárraga J, Huerta-Cepas J, Burguet J, Vaquerizas JM, Conde L, Minguez P, Vera J, Mukherjee S, Valls J, Pujana MA, Alloza E, Herrero J, Al-Shahrour F, Dopazo J: Next station in microarray data analysis: GEPAS. Nucleic Acids Res 2006, 34(Web Server issue):W486-W491
19. Reich M, Ohm K, Angelo M, Tamayo P, Mesirov JP: GeneCluster 2.0: an advanced toolset for bioarray analysis. Bioinformatics 2004, 20: $1797-1798$

20. Morrissey ER, Diaz-Uriarte R: Pomelo II: finding differentially expressed genes. Nucleic Acids Res 2009, 37(Web Server issue): W581-W586

21. Benjamini Y, Drai D, Elmer G, Kafkafi N, Golani I: Controlling the false discovery rate in behavior genetics research. Behav Brain Res 2001, 125:279-284

22. Al-Shahrour F, Diaz-Uriarte R, Dopazo J: FatiGO: a web tool for finding significant associations of Gene Ontology terms with groups of genes. Bioinformatics 2004, 20:578-580

23. Subramanian A, Tamayo P, Mootha VK, Mukherjee S, Ebert BL, Gillette MA, Paulovich A, Pomeroy SL, Golub TR, Lander ES, Mesirov JP: Gene set enrichment analysis: a knowledge-based approach for interpreting genome-wide expression profiles. Proc Natl Acad Sci USA 2005, 102:15545-15550

24. Dolcet X, Llobet D, Encinas M, Pallares J, Cabero A, Schoenenberger JA, Comella JX, Matias-Guiu X: Proteasome inhibitors induce death but activate NF-kappaB on endometrial carcinoma cell lines and primary culture explants. J Biol Chem 2006, 281:22118-22130

25. Cuccuru G, Lanzi C, Cassinelli G, Pratesi G, Tortoreto M, Petrangolini G, Seregni E, Martinetti A, Laccabue D, Zanchi C, Zunino F: Cellular effects and antitumor activity of RET inhibitor RPI-1 on MEN2A-associated medullary thyroid carcinoma. J Natl Cancer Inst 2004, 96:1006-1014

26. Gorla L, Mondellini P, Cuccuru G, Miccichè F, Cassinelli G, Cremona M, Pierotti MA, Lanzi C, Bongarzone I: Proteomics study of medullary thyroid carcinomas expressing RET germ-line mutations: identification of new signaling elements. Mol Carcinog 2009, 48:220-231

27. Gallel P, Pallares J, Dolcet X, Llobet D, Eritja N, Santacana M, Yeramian A, Palomar-Asenjo V, Lagarda H, Mauricio D, Encinas M, Matias-Guiu X: Nuclear factor-kappaB activation is associated with somatic and germ line RET mutations in medullary thyroid carcinoma. Hum Pathol 2008, 39:994-1001

28. Ameur N, Lacroix L, Roucan S, Roux V, Broutin S, Talbot M, Dupuy C, Caillou B, Schlumberger M, Bidart JM: Aggressive inherited and sporadic medullary thyroid carcinomas display similar oncogenic pathways. Endocr Relat Cancer 2009, 16:1261-1272

29. Deng Y, Nagae G, Midorikawa Y, Yagi K, Tsutsumi S, Yamamoto S, Hasegawa K, Kokudo N, Aburatani H, Kaneda A: Identification of genes preferentially methylated in hepatitis $\mathrm{C}$ virus-related hepatocellular carcinoma. Cancer Sci 2010, 101:1501-1510

30. Zacharias A, Gage PJ: Canonical Wnt/beta-catenin signaling is required for maintenance but not activation of Pitx2 expression in neural crest during eye development. Dev Dyn 2010, 239:3215-3225

31. Steiner P, Kulangara K, Sarria JCF, Glauser L, Regazzi R, Hirling H: Reticulon 1-C/neuroendocrine-specific protein-C interacts with SNARE proteins. J Neurochem 2004, 89:569-580

32. Ohiwa M, Murakami H, Iwashita $\mathrm{T}$, Asai $\mathrm{N}$, Iwata $\mathrm{Y}$, Imai $\mathrm{T}$, Funahashi H, Takagi H, Takahashi M: Characterization of Ret-Shc-Grb2 complex induced by GDNF, MEN 2A, and MEN 2B mutations. Biochem Biophys Res Commun 1997, 237:747-751

33. Schlumberger M, Carlomagno F, Baudin E, Bidart JM, Santoro M: New therapeutic approaches to treat medullary thyroid carcinoma. Nat Clin Pract End Met 2008, 4:22-32

34. Khurana R, Agarwal A, Bajpai VK, Verma N, Sharma AK, Gupta RP, Madhusudan KP: unraveling the amyloid associated with human medullary thyroid carcinoma. Endocrinology 2004, 145: $5465-5470$

35. Watanabe T, Ichihara M, Hashimoto M, Shimono K, Shimoyama Y, Nagasaka T, Murakumo Y, Murakami H, Sugiura H, Iwata H, Ishiguro N, Takahashi M: Characterization of gene expression induced by RET with MEN2A or MEN2B mutation. Am J Pathol 2002, 161: 249-256

36. Musholt T, Hanack J, Brehm C, von Wasielewski R, Musholt PB: Searching for non-RET molecular alterations in medullary thyroid 
carcinoma: expression analysis by mRNA differential display. World $\mathbf{J}$ Surg 2005, 29:472-482

37. Lacroix L, Lazar V, Michiels S, Ripoche H, Dessen P, Talbot M, Caillou B, Levillain JP, Schlumberger M, Bidart JM: Follicular thyroid tumors with the PAX8-PPARgamma1 rearrangement display characteristic genetic alterations. Am J Pathol 2005, 167:223-231

38. Jain S, Watson MA, DeBenedetti MK, Hiraki Y, Moley JF, Milbrandt J: Expression profiles provide insights into early malignant potential and skeletal abnormalities in multiple endocrine neoplasia type 2B syndrome tumors. Cancer Res 2004, 64:3907-3913

39. Tofighi R, Joseph B, Xia S, Xu ZQ, Hamberger B, Hökfelt T, Ceccatelli S: Galanin decreases proliferation of PC12 cells and induces apoptosis via its subtype 2 receptor (GalR2). Proc Natl Acad Sci USA 2008, 105:2717-2722

40. Berger A, Santic R, Hauser-Kronberger C, Schilling FH, Kogner P, Ratschek M, Gamper A, Jones N, Sperl W, Kofler B: Galanin and galanin receptors in human cancers. Neuropeptides 2005, 39: 353-359

41. Hawes JJ, Narasimhaiah R, Picciotto MR: Galanin and galanin-like peptide modulate neurite outgrowth via protein kinase C-mediated activation of extracellular signal-related kinase. Eur J Neurosci 2006, 23:2937-2946

42. Sugimoto T, Seki N, Shimizu S, Kikkawa N, Tsukada J, Shimada H, Sasaki K, Hanazawa T, Okamoto Y, Hata A: The galanin signaling cascade is a candidate pathway regulating oncogenesis in human squamous cell carcinoma. Genes Chromosomes Cancer 2009, 48: 132-142

43. Machens A, Brauckhoff M, Holzhausen HJ, Thanh PN, Lehnert H, Dralle H: codon-specific development of pheochromocytoma in multiple endocrine neoplasia type 2. J Clin Endocrinol Metab 2005, 90:3999-4003

44. Gimm O, Dziema H, Brown J, Hoang-Vu C, Hinze R, Dralle H, Mulligan LM, Eng C: Over-representation of a germline variant in the gene encoding RET co-receptor GFR alpha-1 but not GFR alpha-2 or
GFR alpha-3 in cases with sporadic medullary thyroid carcinoma. Oncogene 2001, 20:2161-2170

45. Matsui A, Yamaguchi T, Maekawa S, Miyazaki C, Takano S, Uetake $\mathrm{T}$, Inoue $\mathrm{T}$, Otaka $\mathrm{M}$, Otsuka $\mathrm{H}$, Sato $\mathrm{T}$, Yamashita $\mathrm{A}$, Takahashi Y, Enomoto N: DICKKOPF-4 and -2 genes are upregulated in human colorectal cancer. Cancer Sci 2009, 100:1923-1930

46. Mao B, Niehrs C: Kremen2 modulates Dickkopf2 activity during Wnt/LRP6 signaling. Gene 2003, 302:179-183

47. Rückert F, Joensson P, Saeger HD, Grützmann R, Pilarsky C: Functional analysis of LOXL2 in pancreatic carcinoma. Int J Colorectal Dis 2010, 25:303-311

48. Peng L, Ran YL, Hu H, Yu L, Liu Q, Zhou Z, Sun YM, Sun LC, Pan J, Sun LX, Zhao P, Yang ZH: Secreted LOXL2 is a novel therapeutic target that promotes gastric cancer metastasis via the Src/FAK pathway. Carcinogenesis 2009, 30:1660-1669

49. Peinado H, Moreno-Bueno G, Hardisson D, Pérez-Gómez E, Santos V, Mendiola M, de Diego JI, Nistal M, Quintanilla M, Portillo F, Cano A: Lysyl oxidase-like 2 as a new poor prognosis marker of squamous cell carcinomas. Cancer Res 2008, 68:4541-4550

50. Peinado H, Del Carmen Iglesias-de la Cruz M, Olmeda D, Csiszar K, Fong KS, Vega S, Nieto MA, Cano A, Portillo F: A molecular role for lysyl oxidase-like 2 enzyme in snail regulation and tumor progression. EMBO J 2005, 24:3446-3458

51. Zhu W, Hai T, Ye L, Cote GJ: Medullary thyroid carcinoma cell lines contain a self-renewing CD133+ population that is dependent on ret proto-oncogene activity. J Clin Endocrinol Metab 2010, 95:439-444

52. Raso A, Mascelli S, Biassoni R, Nozza P, Kool M, Pistorio A, Ugolotti E, Milanaccio C, Pignatelli S, Ferraro M, Pavanello M, Ravegnani M, Cama A, Garrè ML, Capra V: High levels of PROM1 (CD133) transcript are a potential predictor of poor prognosis in medulloblastoma. Neuro Oncol 2011, 13:500-508

53. Cook M, Yu XM, Chen H: Notch in the development of thyroid $\mathrm{C}$-cells and the treatment of medullary thyroid cancer. Am J Transl Res 2010, 2:119-125 